\title{
Cor e Produção de Respostas ao Método de Rorschach em um Grupo de Jovens
}

\section{Color and Production of Reponses to the Rorschach Method in a Group of Young}

\author{
Danilo R. Silva ${ }^{1}$ e Ana Sousa Ferreira ${ }^{2}$
}

\section{Resumo}

Na sequência dos nossos estudos sobre o efeito da cor na produção de respostas ao Rorschach, pretendeu-se abordar o estudo do efeito da cor na qualidade formal das respostas ao Rorschach. Com este objectivo, aplicaram-se os cartões I, II, III, VIII, IX e X, na sua versão padronizada e numa versão acromática, a um grupo de jovens dos 15 aos 21 anos. No estudo que nos ocupa sobre o efeito da cor na produção de respostas (2002, 2009), consideraram-se tão só os três últimos cartões VIII, IX e X, inteiramente coloridos. Os resultados confirmam os anteriormente obtidos (2014), pois revelam uma diferença altamente significativa entre médias, favorável à versão cromática no Cartão X bem como entre as médias dos três cartões coloridos.

Palavras-chave: Rorschach, cor, produção de respostas

\begin{abstract}
In the sequence of our study on the effect of color on the production of responses to Rorschach, it was decided to consider the effect of color on the formal quality of responses to Cards II, III, VIII, IX, and X of Rorschach, in their original and in an achromatic version, with a group of young people aged 15-21 years old. In the current study, we considered only the answers given to the last three colored cards (2002 e 2009). Results confirm those obtained previously (2014), as they reveal a highly significant difference between means, favorable to the chromatic version, in Card X, and in all three cards taken together.
\end{abstract}

Keywords: Rorschach, color, production of responses

\footnotetext{
${ }^{1}$ Membro correspondente da Academia das Ciências de Lisboa, Portugal. Tel.: 217571311. E-mail: danilo@psicologia.ulisboa.pt

${ }^{2}$ Faculdade de Psicologia, Universidade de Lisboa e BRU-Instituto Universitário de Lisboa, Portugal. Tel.: 217943655. E-mail: asferreira@psicologia.ulisboa.pt 


\section{Introdução}

O método de Rorschach consiste na aplicação individual de um conjunto de dez cartões com borrões de tinta, devendo a pessoa, diante de cada um deles, responder, à pergunta "O que poderia ser isto?" (Rorschach, 1967, p. 2). Desses cartões, cinco são acromáticos, com diversas tonalidades escuras, dois apresentam também manchas vermelhas e três são inteiramente coloridos. A identificação de objectos é, geralmente, determinada pelo aspecto formal dos borrões ou manchas de tinta, mas podem sê-lo igualmente pela cor, pelas tonalidades ou pela sugestão de movimento que transmitem. Com base nestes múltiplos determinantes de resposta, na modalidade da respectiva localização, no conteúdo invocado e sua qualidade formal, isto é, grau de adequação da forma à área do borrão indicada, Rorschach (1921) elaborou um método de análise e interpretação que visava, primordialmente, a descrição e caracterização da personalidade da pessoa, método que foi publicado em 1921. Objecto de estudo e reflexão, durante décadas, teve o seu apogeu, como instrumento de avaliação, nos anos cinquenta, não só em alguns países europeus, mas também e sobretudo nos EUA, onde surgiram importantes e respeitáveis sistemas de análise e avaliação.

Com o progressivo sucesso no desenvolvimento dos instrumentos de avaliação da personalidade, designados de questionários e inventários, e da metodologia quantitativa colocada ao serviço da sua construção e utilização, que lhes conferiu a almejada validade no decurso dos anos cinquenta, o método de Rorschach, entretanto integrado por Frank (1939) entre os testes por ele designados de "projectivos" e desprovido daquelas características, veio a ser alvo de fortes críticas e de atitudes despiciendas, ao longo da década de sessenta, por parte dos que apenas confiavam no rigor dos números. A verdade, porém, é que o teste ou método de Rorschach, como é comummente conhecido, nunca deixou de ser usado, designadamente no âmbito da psicologia clínica, nem de ter os seus sequazes e defensores, entre respeitáveis cientistas da Psicologia, como o são, por exemplo, Roy Schafer (1922) e Robert R. Holt (1917).
Foi assim que, nos anos sessenta, surgiu, nos EUA, um autor que decidiu lançar mãos à ingente tarefa de construir um novo sistema que integraria os elementos mais consistentes, geralmente aceites e reconhecidos como dotados de fundamento objectivo, presentes nos cinco sistemas de Rorschach, então vigentes naquele país (Exner, 1969). Esse empreendimento deu origem ao Sistema Integrativo do Rorschach, do seu autor John E. Exner, Jr. (1974), que veio, com o decorrer dos anos e mercê de prolongada e persistente investigação, a ganhar as características de precisão e validade requeridas aos instrumentos de avaliação psicológica. O S.I.R. mantém-se, actualmente, entre os meios mais utilizados nos múltiplos campos daquele ramo da psicologia, seja o normal seja o clínico ou patológico seja o jurídico. Esta linha manteve-se e veio a revelar os seus frutos, após a sua morte em 2006, com a publicação pelos seus colaboradores mais próximos, Meyer, Viglione, Mihura, Erard e Erdberg, em 2011, da obra Rorschach Performance Assessment System (Meyer et al.).

Instrumento de avaliação complexo por inerência, dada a multiplicidade de fontes de informação que o servem ou de que parte, as quais requerem um conhecimento tão amplo e profundo quanto possível, o Rorschach permanece um meio de estudo e investigação aberto. Mostra-o concretamente a linha de investigação que se nos ofereceu, através de uma proposta de John E. Exner, nos anos noventa, em Madrid.

\section{Cor e produção de respostas}

Nos finais dos anos cinquenta e início dos sessenta, Exner havia mostrado, com um grupo de estudantes universitários, que o elemento ou determinante cor, no Rorschach, estava na origem do aumento da produção de respostas. Verificara que o maior número de respostas observado, geralmente, nos três últimos dos dez cartões aplicados, se devia ao facto de serem coloridos (Exner,1962). Antes dele, outro estudioso das características dos cartões do Rorschach e autor de diversos trabalhos nesse campo, não tinha detectado tal pormenor (Baughman, 1959). Acresce que Bruno Klopfer, um dos grandes sistematizadores deste instrumento, afirmava, já nos anos quarenta, que o carácter fragmentado do último dos cartões, o Cartão X, estaria na origem 
do maior número de respostas que suscitava, em comparação com os dois cartões coloridos anteriores menos divididos. A este respeito escreviam Ainsworth e Klopfer, em 1954: "Assim, se mais do que quarenta por cento das respostas forem dadas aos três últimos cartões (contanto que mais do que vinte por cento não sejam dadas apenas ao Cartão X), a hipótese é a de que a produção do indivíduo é estimulada pelo impacto ambiente, quer ele dê expressão manifesta à sua reacção emocional quer não" (p. 297).

Tendo em conta que o Cartão $X$, pela sua estrutura, suscita elevado número de repostas de detalhe, acrescentava Klopfer, aquela hipótese só deverá ser considerada se a soma das respostas aos três cartões for de $40 \%$ ou mais do total das respostas dadas. Salvaguardava, porém, a condição de que se o Cartão X suscitasse mais do que $20 \%$ das respostas dadas aos mesmos três cartões, o significado contido na referida hipótese não seria validado. Esta ideia, com outro fundamento, parece achar-se também presente no Rorschach Performance Assessment System (R$P A S)$, onde, a propósito da variável $R 8910 \%$ percentagem de respostas dadas aos cartões coloridos - se escreve:

"O fundamento teórico deste código é a ideia de que entre as pessoas que são estimuladas por e atraídas para situações emocionais, a produtividade das respostas aumentaria com as cores vivas. Mas a reacção à cor e a elevação do R8910\% reflectem provavelmente processos que não se confinam ao processamento relacionado com a emoção mas estão também relacionados com uma reactividade mais geral aos estímulos fortes, irresistíveis e excitantes. Por outro lado, algumas inconsistências entre o cálculo da variável e o seu processo de resposta são também dignas de nota: o $R 8910 \%$ pode ser enganador em casos com muitas respostas simplistas aos últimos três cartões (mais frequentemente visto como múltiplos códigos "D A 2 F" ao Cartão X), já que o número de tais respostas pode dizer algo mais sobre a dificuldade de integrar estes cartões divididos em perceptos mais extensos do que sobre o impacto estimulante da cor." (Meyer et al., 2011, p. 454)

Ora, o que Exner propunha como objecto de investigação era precisamente verificar se o maior número de respostas, com frequência observado no Cartão X, se devia à sua estrutura dividida, responsável pelo elevado número de respostas de detalhe, ou à sua qualidade cromática.

$\mathrm{Na}$ concretização deste estudo, a metodologia observada consistiu na aplicação dos dez cartões do Rorschach, na sua versão estandardizada e numa versão acromática, criada para o efeito, a dois grupos de vinte jovens de ambos os géneros, tal como havia procedido Exner. Dado o nosso interesse e prática frequente de avaliação de crianças decidiu-se repetir o mesmo estudo com um grupo de crianças de ambos os géneros, com 9/10 anos, já que os estudos do Rorschach com crianças são muito menos frequentes do que com adultos (Silva, 2000/2001, 2002).

Os resultados obtidos confirmaram a hipótese de Exner segundo a qual o maior número de respostas habitualmente observado no Cartão X se deve à qualidade cromática do estímulo e não ao facto de ser dividido ou fragmentado, o que invalida a hipótese de Klopfer e coloca entre parêntesis a posição do R-PAS antes referida. Poderíamos ainda acrescentar que, se a frequência de respostas simplistas fosse elevada, tal dado não permitiria a obtenção clara do resultado que atesta a cor como o elemento responsável pela elevação do número de respostas neste Cartão, face ao observado na sua versão acromática.

Por sua vez, o estudo com o grupo de crianças de 9/10 anos não revelou qualquer efeito da cor no aumento da produção de respostas, um dado decididamente surpreendente, já que é reconhecido o efeito atraente que a cor tem nas crianças, designadamente em certos testes psicológicos, como a Escala de Inteligência de Wechsler para Crianças ou o teste das Matrizes Progressivas Coloridas para crianças. Os autores destes instrumentos de avaliação adoptaram o cromatismo dos estímulos como modo de atracção e sustentação da atenção na execução da tarefa.

Aqui, deparamos com um dado que corrobora a qualidade de abertura do método de Rorschach a nova investigação, inicialmente destacado. Com efeito, apesar dos múltiplos e numerosos estudos sobre a cor e seus efeitos no Rorschach, ainda não se verificara que a cor, nos protocolos de crianças, não só não tem o efeito de aumentar a produção de respostas observado no adulto mas tem mesmo o efeito contrário de a inibir, como o revelaram 
novos estudos (Silva \& Marques, 2008), um dado desenvolvimentista importante até agora ignorado. Este constitui um elemento relevante $\mathrm{e}$ diferenciador da natureza da tarefa que constitui o dar uma resposta ao Rorschach. Enquanto, no subteste dos cubos da WISC e no teste da Matrizes Progressivas Coloridas, a cor tem esse efeito de atrair e prender a atenção do testando mas não é decisiva no acto de dar a resposta, no Rorschach a presença da cor constitui parte do problema equacionado pela pergunta "O que poderia ser isto?", como muito bem o salientou Exner, ao caracterizar a resposta ao Rorschach como uma solução do problema posto por este modo de avaliação.

A particularidade do efeito inibitório da cor na produção de respostas, a partir dos $5 / 6$ anos até aos 11/12 anos, acha-se bem confirmada pelos dados normativos de crianças. Dois exemplos o ilustram de modo claro: o de Exner e Weiner (1995), com crianças dos 5 aos 16 anos, onde se verifica uma redução do Quociente Afectivo ( $A f r$ ), expresso pela divisão do número de respostas dadas aos cartões VIII, IX e X pelo número de respostas dadas aos restantes cartões, o qual desce de .82 , nos 5 anos, para .62 nos 12 anos; e os dados normativos portugueses para crianças dos 6 aos 10 anos, onde a redução do Quociente Afectivo é menos acentuada e regular do que a norte-americana mas reconhecível, .59, .53, .51, .52 e .55 , como paralelamente o é o aumento do número médio de respostas dadas aos restantes seis cartões que sobe das 9 respostas, nos 6 anos, para 12, nos 7, 8 e 9 anos, e 11 nos 10. (Silva \& Dias, 2007). O facto de, apenas aos $15 / 16$ anos, se verificar o aumento da produção de respostas, como efeito da cor, constitui um dado desenvolvimentista importante, pois, só a partir deste período etário, o significado das variáveis que assentam na cor, tais o Quociente Afectivo (Afr) (Exner, 2003) ou RC\% (Klopfer, 1954, e a escola francesa) ou R8910\% (R-PAS) e as respostas cor $(\mathrm{FC}, \mathrm{CF}, \mathrm{C})$ adquirem o significado que lhes é atribuído no Rorschach de adultos.

É, no entanto, oportuno salientar que este dado, comprovado pelos estudos referidos, não se verifica em todas as populações e culturas, isto é, não deve ser considerado universal. Com efeito, um estudo efectuado na cidade moçambicana de Quelimane revelou que, num grupo de crianças de ambos os sexos e idades de 11/12 anos, a cor, tal como foi observado em Lisboa, não tem o efeito de aumentar a produção de respostas, mas idêntica ausência se observa também num grupo de adolescentes de ambos os sexos, com idades entre os 15 e os 20 anos, diversamente do verificado em Lisboa ou nos EUA (Silva \& Ferreira, 2011).

\section{Estudo com jovens}

O presente estudo, diferentemente dos anteriormente referidos sobre idêntico tema, integrou-se em um outro que se propôs verificar em que medida a cor pode ser responsável pela maior frequência de respostas de distorção perceptiva, isto é, com qualidade formal desadequada, verificada nos estudos normativos de adultos americano (Exner \& Erdberg, 2005), português (Pires, 2007) e normativo português de crianças (Silva \& Dias, 2007). Com efeito, naquele estudo, foram aplicados, além dos cartões coloridos, VIII, IX e X, os cartões II e III, onde o vermelho se junta aos tons escuros.

\section{Participantes}

A amostra é constituída por 80 adolescentes, 40 do sexo masculino e 40 do sexo feminino, com idades entre os 15 e os 21 anos (ver Quadro 1), provenientes de quatro escolas secundárias do Funchal: Ângelo Augusto da Silva (AAS), Gonçalves Zarco (GZ), Francisco Franco (FF) e Liceu de Jaime Moniz (L) (ver Quadro 2).

Quadro 1. Idades e percentagem de respondentes

\begin{tabular}{lccc}
\multicolumn{4}{c}{ por grupo $(\mathrm{N}=80)$} \\
\hline Idades & Respondentes & $\begin{array}{c}\text { Frequência } \\
\text { relativa } \\
\text { percentual }\end{array}$ & $\begin{array}{c}\text { Frequência } \\
\text { relativa } \\
\text { percentual } \\
\text { acumulada }\end{array}$ \\
\hline $15 / 16$ & 10 & 13 & 13 \\
17 & 48 & 60 & 73 \\
18 & 13 & 16 & 89 \\
$19 / 21$ & 9 & 11 & 100 \\
\hline
\end{tabular}

Quadro 2. Escolas, frequência e percentagem dos adolescentes examinados

\begin{tabular}{lccc}
\hline Escolas* & Frequência & $\begin{array}{c}\text { Frequência } \\
\text { relativa } \\
\text { percentual }\end{array}$ & $\begin{array}{c}\text { Frequência } \\
\text { relativa } \\
\text { percentual } \\
\text { acumulada }\end{array}$ \\
\hline AAS & 24 & 30.0 & 30.0 \\
GZ & 20 & 25.0 & 55.0 \\
FF & 17 & 21.3 & 76.3 \\
L & 19 & 23.8 & 100.0 \\
Total & 80 & 100.0 &
\end{tabular}

*Nota. AAS - Ângelo Augusto da Silva; GZ - Gonçalves Zarco; FF - Francisco Franco; L - Liceu de Jaime Moniz 


\section{Método}

$\mathrm{O}$ primeiro momento do planeamento do trabalho consistiu na obtenção da necessária autorização dos Serviços de Educação da Madeira bem como das respectivas Escolas e encarregados de educação dos adolescentes que aceitassem ser voluntários nesta investigação.

Os cartões VIII, IX e X foram aplicados nesta mesma sequência, após os cartões I, II e III, nas suas versões cromática e acromática, tendo o Cartão I, acromático, sido aplicado com o intuito apenas metodológico de preparar o respondente para o tipo de tarefa proposta. As instruções adoptadas foram as indicadas por Exner na aplicação do Rorschach: O que poderia ser isto?

A exploração dos resultados tomou em consideração as variáveis cromático/acromático, sexo e escola, tendo em vista verificar até que ponto estas outras duas últimas variáveis exerciam alguma influência na frequência de respostas ao carácter cromático ou acromático dos diferentes cartões. Para isso, procedeu-se a uma Análise de Variância a dois factores (cromático/acromático e sexo) tendo em vista o número de respostas a cada um dos cartões VIII, IX e X e o conjunto dos três; e a uma Análise de Variância a um factor (escola), com idêntico âmbito, tendo em conta a característica cromático/acromático. Esta exploração esteve a cargo do segundo autor.

\section{Análise dos resultados}

O Quadro 3 apresenta os resultados da Análise de Variância a dois factores - sexo e cromático/acromático - em cada um dos cartões VIII, IX e $\mathrm{X}$ e no conjunto dos três. Se observarmos os resultados obtidos com o Cartão VIII, verificamos que não se detectam diferenças significativas no número de respostas por nenhuma das variáveis, sexo e cromático/acromático, nem na respectiva interacção. Com efeito, o número médio de respostas obtidas é bastante próximo, quer quando se considera a variável sexo quer quando se considera a variável cromático/acromático ou a interacção entre as duas. Os valores da potência de teste são em todos os casos baixos. Vale a pena registar que, embora as diferenças entre médias não sejam significativas, os respectivos valores são mais elevados no sexo feminino e nos cartões cromáticos do que no masculino e nos acromáticos.

No Cartão IX, obtêm-se resultados semelhantes aos obtidos no Cartão anterior. Não se observa nenhum efeito significativo de qualquer das vartiáveis ou da sua interacção na produção de respostas.

No Cartão X, a Análise de Variância revela uma diferença altamente significativa na produção de respostas, com valor de teste muito elevado, na versão cromática/acromática, favorável à versão cromática. Nem a variável sexo nem a interacção entre as duas variáveis consideradas revelam qualquer influência significativa na produção de respostas a este Cartão. Este dado não deixa de merecer a devida consideração, face à longa citação acima extraída do RPAS relativa à variável R8910\%. Nos três estudos por nós efectuados anteriormente, com amostras de ambos os sexos e idades a partir dos 15 anos, verificou-se um aumento significativo da produção de respostas à versão cromática do Cartão $\mathrm{X}$, confirmando o papel dominante da cor na ocorrência desse dado. Com efeito, poderá pensarse que, se o factor determinante de resposta fosse a facilidade para dar respostas de detalhe, isso ocorreria tanto na versão cromática como na acromática e não haveria lugar para uma diferença significativa como a ocorrida.

Quando se considera o número de respostas no conjunto dos três cartões, verifica-se a ocorrência de uma diferença altamente significativa, com poder de teste elevado, entre as versões cromática/acromática, favorável ao carácter cromático dos cartões, ou seja, tomado o número total das respostas dadas aos cartões na sua versão cromática e acromática, a versão cromática dá lugar a uma média de respostas significativamente mais alta do que a da versão acromática. Assim, se atentarmos aos dados obtidos, podem observar-se dois aspectos constantes: (1) um, principal, que se traduz no facto de, nos três cartões, o valor médio, obtido no factor cromático/acromático, ser sempre superior na coluna cromático do que na coluna acromático; (2) um aspecto secundário que se traduz pela circunstância de o sexo feminino obter sempre um 
Quadro 3. Resultados da análise de variância a dois factores (cromático/acromático e sexo) para o número de respostas aos Cartões VIII, IX e X e no conjunto dos três cartões

\begin{tabular}{|c|c|c|c|c|c|c|c|c|}
\hline Cartão & Factores & \multicolumn{2}{|c|}{$\begin{array}{c}\text { Estatísticas Descritivas } \\
\text { M (DP) }\end{array}$} & \multirow[t]{2}{*}{$\begin{array}{c}\text { Estatística } \\
\text { F }\end{array}$} & \multirow[t]{2}{*}{ g.l. } & \multirow[t]{2}{*}{ Sig. } & \multirow[t]{2}{*}{$\eta^{2}$} & \multirow[t]{2}{*}{$\begin{array}{r}\text { Potênci } \\
\text { de teste }\end{array}$} \\
\hline \multirow{9}{*}{$\begin{array}{l}\text { Cartão } \\
\text { VIII }\end{array}$} & & Masculino & Feminino & & & & & \\
\hline & Sexo & $2.60(0.90)$ & $2.77(0.83)$ & .81 & 1 & .37 & .01 & .15 \\
\hline & & Cromático & Acromático & & & & & \\
\hline & Crom/Acrom & $2.80(0.99)$ & $2.57(0.71)$ & 1.34 & 1 & .25 & .02 & .21 \\
\hline & & Crom & Acrom & & & & & \\
\hline & & $\mathrm{M} \quad \mathrm{F}$ & $\mathrm{M} \quad \mathrm{F}$ & & & & & \\
\hline & Sexo*Crom/acrom & $\begin{array}{cc}2.65 & 2.95 \\
(1.04) & (0.95)\end{array}$ & $\begin{array}{cc}2.55 & 2.60 \\
(0.76) & (0.68)\end{array}$ & .42 & 1 & .52 & .01 & .10 \\
\hline & & Masculino & Feminino & & & & & \\
\hline & Sexo & $2.43(1.08)$ & $2.75(1.03)$ & 1.87 & 1 & .18 & .02 & .27 \\
\hline \multirow{4}{*}{$\begin{array}{l}\text { Cartão } \\
\text { IX }\end{array}$} & & Cromático & Acromático & & & & & \\
\hline & Crom/Acrom & 2.70 & $2.48(0.96)$ & .90 & 1 & .35 & .01 & .16 \\
\hline & & Crom & Acrom & & & & & \\
\hline & Sexo*Crom/Acrom & $\begin{array}{cc}\mathrm{M} & \mathrm{F} \\
2.45 & 2.95 \\
(1.10) & (1.19)\end{array}$ & \begin{tabular}{cl}
$\mathrm{M}$ & \multicolumn{1}{c}{$\mathrm{F}$} \\
2.40 & 2.55 \\
$(1.10)$ & $(0.83)$
\end{tabular} & .54 & 1 & .46 & .01 & 0.11 \\
\hline \multirow{9}{*}{$\begin{array}{l}\text { Cartão } \\
\text { X }\end{array}$} & & Masculino & Feminino & & & & & \\
\hline & Sexo & $\begin{array}{c}3.98 \\
(1.29)\end{array}$ & $\begin{array}{c}4.18 \\
(1.20)\end{array}$ & .63 & 1 & .43 & .00 & .12 \\
\hline & & Cromático & Acromático & & & & & \\
\hline & Crom/acrom & $\begin{array}{c}4.63 \\
(1.21)\end{array}$ & $\begin{array}{c}3.53 \\
(1.01)\end{array}$ & 19.06 & 1 & $.00 * *$ & .20 & .99 \\
\hline & & Cromático & Acromático & & & & & \\
\hline & & $\mathrm{M} \quad \mathrm{F}$ & $\mathrm{M} \quad \mathrm{F}$ & & & & & \\
\hline & Sexo*Crom/Acrom & $\begin{array}{cc}4.55 & 4.70 \\
(1.23) & (1.22)\end{array}$ & $\begin{array}{cc}3.40 & 3.65 \\
(1.10) & (0.93)\end{array}$ & .04 & 1 & .84 & .00 & .05 \\
\hline & & Masculino & Feminino & & & & & \\
\hline & Sexo & $\begin{array}{c}9.00 \\
(2.62)\end{array}$ & $\begin{array}{l}9.70 \\
(2.05)\end{array}$ & 1.94 & 1 & .17 & .03 & .28 \\
\hline \multirow{4}{*}{$\begin{array}{l}\text { VIII+ } \\
\text { IX+X }\end{array}$} & & Cromático & Acromático & & & & & \\
\hline & Crom/Acrom & $\begin{array}{c}10.13 \\
(2.44) \\
\text { Cromático }\end{array}$ & $\begin{array}{c}8.58 \\
(2.04) \\
\text { Acromático }\end{array}$ & 9.53 & 1 & $.00 * *$ & .11 & .86 \\
\hline & & $\mathrm{M} \quad \mathrm{F}$ & $\mathrm{M} \quad \mathrm{F}$ & & & & & \\
\hline & Sexo*Crom/Acrom & $\begin{array}{cc}9.65 & 10.60 \\
(2.72) & (2.09) \\
\end{array}$ & $\begin{array}{cc}8.35 & 8.80 \\
(2.41) & (1.61)\end{array}$ & .25 & 1 & .62 & .00 & .08 \\
\hline
\end{tabular}

número médio de respostas ligeiramente mais elevado do que o do masculino, muito embora esse nível nunca seja significativamente maior nem mesmo quando se consideram os três cartões juntos.

O Quadro 4 apresenta os resultados da Análise de Variância a um factor - escola - para o número de respostas dadas aos Cartões VIII, IX e $\mathrm{X}$ e no conjunto dos três cartões, na versão cromática e acromática.

A análise dos resultados por Cartão revela que, para o Cartão VIII, se verifica que, entre os adolescentes das escolas AAS e GZ, onde foi aplicada a versão acromática a 20 adolescentes, se observa uma diferença significativa entre o número médio de respostas dadas favorável à escola AAS. Tal não se observa entre as escolas onde foi aplicada a versão cromática.

Nos Cartões IX e X, não ocorrem diferenças significativas, muito embora se possa observar que os adolescentes da escola AAS obtêm, em ambos, um número médio mais alto do que a escola GZ. Este dado contribui, seguramente, para que, no conjunto dos três cartões, ocorra, de novo, uma diferença significativa entre aquelas escolas, ao nível de significância de 0,05 , superior ao de .00 registado no Cartão VIII. 
Quadro 4. Resultados das análises de variância a um factor para o número de respostas nos Cartões VIII, IX e X por escola no caso cromático e no caso acromático

\begin{tabular}{|c|c|c|c|c|c|c|c|c|c|c|}
\hline Cartão & Crom/Acrom & Escola & $\mathrm{N}$ & Média & DP & $\mathrm{F}$ & g.l. & Sig. & $\eta^{2} p$ & $\begin{array}{c}\text { Potência de } \\
\text { teste }\end{array}$ \\
\hline \multirow{7}{*}{ VIII } & \multirow{3}{*}{ Cromático } & AAS & 4 & 3.00 & 1.16 & & & & & \multirow{3}{*}{.07} \\
\hline & & FF & 17 & 2.82 & 1.02 & \multirow{2}{*}{.12} & \multirow{2}{*}{2} & \multirow{2}{*}{.89} & \multirow{2}{*}{.01} & \\
\hline & & $\mathrm{L}$ & 19 & 2.74 & .99 & & & & & \\
\hline & \multirow{3}{*}{ Acromático } & AAS & 20 & 2.85 & .81 & \multirow{3}{*}{6.86} & \multirow{3}{*}{1} & \multirow{3}{*}{$.00 * *$} & \multirow{3}{*}{.15} & \\
\hline & & GZ & 20 & 2.30 & .47 & & & & & \\
\hline & & AAS & 4 & 3.00 & .82 & & & & & \\
\hline & \multirow[t]{2}{*}{ Cromático } & $\mathrm{FF}$ & 17 & 2.76 & 1.30 & \multirow[t]{2}{*}{.25} & \multirow[t]{2}{*}{2} & \multirow[t]{2}{*}{.78} & \multirow[t]{2}{*}{.01} & \multirow[t]{2}{*}{.09} \\
\hline \multirow{3}{*}{ IX } & & $\mathrm{L}$ & 19 & 2.58 & 1.12 & & & & & \\
\hline & \multirow{3}{*}{ Acromático } & AAS & 20 & 2.70 & 1.13 & \multirow{2}{*}{2.27} & \multirow{2}{*}{1} & \multirow{2}{*}{.14} & \multirow{2}{*}{.06} & \multirow{2}{*}{.31} \\
\hline & & GZ & 20 & 2.25 & .72 & & & & & \\
\hline \multirow{7}{*}{$\mathrm{X}$} & & AAS & 4 & 5.00 & .00 & \multirow{3}{*}{.24} & \multirow{3}{*}{2} & \multirow{3}{*}{.79} & \multirow{3}{*}{.01} & \multirow{3}{*}{.08} \\
\hline & \multirow{2}{*}{ Cromático } & $\mathrm{FF}$ & 17 & 17 & 1.07 & & & & & \\
\hline & & $\mathrm{L}$ & 19 & 4.63 & 1.46 & & & & & \\
\hline & \multirow{3}{*}{ Acromático } & AAS & 20 & 3.65 & .99 & 60 & 1 & 44 & 02 & 12 \\
\hline & & GZ & 20 & 3.40 & 1.05 & .00 & 1 & 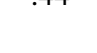 & .02 & .12 \\
\hline & & AAS & 4 & 11.00 & 1.83 & & & & & \\
\hline & Cromático & $\mathrm{FF}$ & 17 & 10.12 & 2.50 & .30 & 2 & .75 & .02 & .09 \\
\hline & & $\mathrm{L}$ & 19 & 9.95 & 2.57 & & & & & \\
\hline $\begin{array}{c}\text { VIII+IX+ } \\
\quad X\end{array}$ & Acromático & AAS & 20 & 9.20 & 2.35 & 406 & 1 & & 10 & \\
\hline & & $\mathrm{GZ}$ & 20 & 7.95 & 1.47 & 4.06 & 1 & $.05^{*}$ & .10 & \\
\hline
\end{tabular}

Estes resultados surgem, no contexto do presente estudo, como destituídos de interesse não só porque nada nos dizem sobre o efeito da cor na produção de respostas como também não afectam a utilização do Rorschach como instrumento de avaliação, pois está em causa a versão acromática de um cartão originalmente cromático. A única informação que nos proporciona é a de que os adolescentes da escola A. A. S. dão um maior número de respostas à versão acromática dos cartões do que os da escola G. Z., o que pode traduzir maior disponibilidade para a tarefa ou capacidade de análise e elaboração dos primeiros.

\section{Discussão}

A análise dos resultados obtidos, referentes à produção de respostas aos cartões do Rorschach ditos coloridos, VIII, IX e X, por uma amostra de 80 adolescentes de ambos os sexos, revelou que, de entre os três factores considerados - cromático cromático/acromático, sexo e escola frequentada apenas o factor cromático/acromático determina a ocorrência de uma diferença altamente significativa no número médio de respostas ao Cartão X, favorável à versão cromática. Nos cartões VIII e IX, não se verifica qualquer efeito significativo dos factores considerados, embora os resultados obtidos sejam mais elevados na versão cromática do que na acromática. A mesma análise revela ainda que, ao considerarem-se os resultados obtidos no conjunto dos três cartões, se identifica, de novo, uma produção de respostas significativamente mais elevada na sua versão cromática. Este resultado reforça, manifestamente, os resultados obtidos nos estudos anteriores (Silva, 2002, Silva \& Ferreira, 2014) e destaca, em particular, a importância da cor na produção de respostas, significativamente mais elevada, no Cartão X. Este último dado reveste-se de particular relevância porquanto põe em causa a hipótese de Bruno Klopfer, para um número muito 
elevado de respostas ao Cartão $\mathrm{X}$, anulador do significado interpretativo da variável VIII $+\mathrm{IX}+\mathrm{X} \%$, bem como a insistência expressa no recente sistema R-PAS acerca da presença de um frequente número de respostas simplistas que, por assim dizer, põem em causa o significado, geralmente atribuído à variável R8910\%, de traduzir a "ideia de que, entre as pessoas que são estimuladas e atraídas por situações emocionais, a produção de respostas deve ser aumentada pelas cores vivas" (Meyer et al, p. 454). Ora, os estudos efectuados confirmam que os cartões coloridos determinam um aumento significativo na produção de respostas. Por outro lado, a afirmação que refere a frequência da resposta simplista "D A 2 F" parece-nos carecer de verificação, pois, no presente estudo, em que se obtiveram, em 39 protocolos, 189 respostas ao Cartão $\mathrm{X}$, versão cromática, 28 ou seja15\% das respostas apresentavam aquela codificação e apenas cinco dos 39 dos protocolos ou 13\% apresentavam um número superior a 1 daquelas respostas: três protocolos com duas e dois com três "D A 2 F". Face a estes dados, parece-nos excessivo considerar o Cartão X como gerador de respostas simplistas, a menos que esta característica se estenda a outros tipos de resposta, muito embora também se tenha presente o reduzido número de protocolos em presença.

Tais resultados têm, no entanto, a particularidade de se distanciarem dos obtidos nos estudos anteriores, já que nestes se verifica uma produção de respostas significativamente mais elevada a cada um dos três cartões coloridos, em comparação com a obtida com a versão acromática dos mesmos.

Procurando uma explicação plausível para este dado e admitindo como seguro que uma diferença cultural em relação aos adolescentes de Lisboa não pode justificar o distanciamento dos resultados encontrados, ponderámos a possibilidade de essa explicação residir na alteração metodológica introduzida em relação aos estudos acima referidos. Com efeito, naqueles, usaram-se esquemas diferentes de aplicação. No de 2002, aplicaram-se os dez cartões do Rorschach, na versão padrão e na versão acromática, segundo a ordem comum e na ordem inversa; no de 2014, aplicaram-se apenas os cartões coloridos na versão padrão e na versão acromática. No caso presente, como foi referido antes, a aplicação dos cartões coloridos foi precedida da dos Cartões II e III ${ }^{\text {bb }}$. Ora acontece que estes cartões, designados, por vezes, de vermelhos, contam-se entre os que suscitam, em alguns estudos normativos, maior número de respostas com má qualidade formal (Silva \& Dias, $2007^{\mathrm{a}}$, Silva \& Dias, 2007 , Pires, 2007, Exner \& Erdberg, 2005). Este dado permite considerar a ideia de que tais cartões ocasionam algum efeito perturbador no funcionamento perceptivocognitivo dos examinados que, eventualmente, se repercute na produção de respostas aos cartões seguintes, neste caso os cartões coloridos VIII, IX e $X$, determinando uma redução do número de respostas dadas. Se assim for, poderá pensar-se que, no caso da aplicação normal do Rorschach, a presença dos cartões acromáticos IV, V, VI e VII, onde o número de respostas FQ- é geralmente menos elevado, tem uma função reparadora da perturbação gerada pelos Cartões II e III, suscitando a ocorrência do aumento da produção de respostas aos três cartões coloridos.

Nestas circunstâncias, impõe-se a repetição do estudo com uma amostra de jovens, com o fim de apurar a consistência da hipótese aqui delineada.

\section{Conclusão}

A aplicação dos Cartões VIII, IX e X do Rorschach, na sua versão cromática e numa versão acromática, a um grupo de jovens revelou que a cor determina o aumento da produção de respostas nos três cartões, significativamente no Cartão $X$ e na soma das respostas dadas ao conjunto dos três cartões. Este resultado invalida, por um lado, o juízo de que tal aumento se deve ao carácter dividido do Cartão X e, por outro, põe em questão a ideia expressa no R-PAS segundo a qual a presença de respostas simplistas denuncia mais a dificuldade de integração das partes em perceptos mais amplos do que o incitamento da cor à resposta. $\mathrm{O}$ facto de os resultados obtidos se distanciarem dos proporcionados pelos estudos anteriores poderá ser efeito de a aplicação dos cartões coloridos, ter sido precedida, no caso presente, da dos Cartões II e III. 


\section{Referências}

Exner, J. E. (1962). The Effect of colour on productivity in cards VIII, IX, $\mathrm{X}$ of the Rorschach. Journal of Projective Techniques, 26, 30-33.

Exner, J. E., \& Weiner, I. B. (1995). The Rorschach: A comprehensive system volume 3: Assessment of children and adolescents, Vol. 2, 2nd edition. New York: John Wiley \& Sons.

Exner, J. E. (2003). The Rorschach: A comprehensive system basic foundations and principles of interpretation Vol 1 (4th edition), New Jersey, John Wiley \& Sons.

Exner, J. E., \& Erdberg, P., (2005). The Rorschach: A comprehensive system. Volume 2 (3rd edition). New Jersey, John Wiley \& Sons.

Frank, L. K. (1939). Projective methods for the study of personality. Journal of Psychology, 8, 389-413.

Meyer, G. J., Viglione, D. J., Mihura, J. L., Erard, R. E., \& Erdberg, P. (2011). Rorschach performance assessment system. administration, coding, interpretation, and technical manual. Published by Rorschach Performance Assessment System, LLC, 2011.

Pires, A. A. (2007). Rorschach comprehensive system data for a sample of 309 nonpatient adults from Portugal. Journal of Personality Assessment, 89, Supplement (S1), S124-S130.

Rorschach, H. (1967). Psychodiagnostic Méthode et résultats d'une experience diagnostique de perception $4^{e}$ edition française révisée et corrigée avec une bibliographie complete et mise à jour par Cécile BEIZMANN. Paris, Presses Universitaires de France.

Silva, D. R. (2000/2001). O efeito da cor na produção de respostas no Rorschach. Revista Portuguesa de Psicologia, 35, 27-44.

Silva, D. R. (2002). The effect of color on productivity on card $\mathrm{X}$ of the Rorschach. Rorschachiana, 25, 123-138.

Silva, D. R. (2009). A Função da cor na produção de respostas ao Rorschach até ao final da adolescência. Revista Iberoamericana de Diagnóstico y Evaluación - e Avaliação Psicológica, 28, 149-165.
Silva, D. R. (2011). Role of color on the production of responses in children's Rorschach protocols. Rorschachiana, 32 (2), 183-198.

Silva, D. R., \& Dias, A. M. (2007). A propósito de la elaboración de una Tabla de Calidad Formal del Rorschach de una muestra de niños portugueses. Revista de la Sociedad Española del Rorschach y Métodos Proyectivos, 20, 34-38.

Silva, D. R., \& Dias, A. M. ${ }^{\text {a }}$ (2007). Rorschach comprehensive system data for a sample of 359 portugese children at five ages. Journal of Personality Assessment, 89(S1), 131-141.

Silva, D. R., \& Marques, L, G. (2008). A cor e o efeito inibitório de respostas no Rorschach de crianças. Psychologica, 48, 5-24.

Silva, D. R., \& Marques, L. G. (2008a). L'effet de la couleur sur la production de réponses au Rorschach chez des enfants non consultants. Une étude génétique. Psychologie Clinique et Projective, 14, 221-232.

Silva, D. R., \& Ferreira, A. S. (2011a). Cor e produção de respostas ao Rorschach: Um estudo transcultural. Paideia, (Ribeirão Preto), Ago 2011, vol. $N^{\circ} 49$, p.149-156.

Silva, D. R., \& Ferreira, A. S. (2014). The effect of color on the production of responses to Rorschach cards VIII, IX, and X in age groups of 11-12 and 15-16 years. Journal of Personality Assessment, 96 (4), 426-431. 